\title{
ABSORBING BOUNDARY CONDITIONS FOR THE LINEARIZED EULER EQUATIONS IN 2-D
}

\author{
DIETMAR KRÖNER
}

\begin{abstract}
In this paper we shall derive some approximate absorbing boundary conditions for the initial value problem for the unsteady linearized Euler equations in 2-D. Since we assume that the coefficients of the system are constant, we can describe the transformation of the system to a decoupled system of ODE's and the related absorbing boundary conditions explicitly. We shall verify the usefulness of these boundary conditions in some numerical tests for the nonlinear Euler equations in 2-D.
\end{abstract}

\section{INTRODUCTION}

When solving numerically an initial value problem for a hyperbolic system in the whole space $R^{2}$ or $R^{3}$, it is necessary to introduce artificial boundaries in order to obtain a finite computational domain. Then the question arises which kind of boundary conditions one has to impose on these additional boundaries. They should guarantee that the solution is not significantly disturbed by reflection or other effects which come from these artificial boundaries. Furthermore, the solution on the finite computational domain should be a good approximation to the solution of the initial value problem in the whole space.

Using the theory of pseudodifferential operators, Engquist and Majda [2] developed the concept of absorbing boundary conditions to satisfy the above requirements. They treated the wave equation and first-order symmetric systems in 2-D. In more detail, they considered the $3 \times 3$ system of the linearized shallow water problem.

In this paper we consider general symmetrizable linear systems of first order, and in particular we treat the $4 \times 4$ system of the linearized Euler equations in primitive variables:

$$
\partial_{t} U+B_{1} \partial_{x} U+B_{2} \partial_{y} U=F
$$

Received April 16, 1990.

1980 Mathematics Subject Classification (1985 Revision). Primary 35L45, 35L65, 65N05, $65 \mathrm{M} 05,76 \mathrm{~N} 15$. 
where (see [9])

$$
\begin{gathered}
U=\left(\begin{array}{c}
\rho \\
u_{1} \\
u_{2} \\
p
\end{array}\right), \quad B_{1}=\left(\begin{array}{cccc}
u_{1} & \rho & 0 & 0 \\
0 & u_{1} & 0 & \frac{1}{\rho} \\
0 & 0 & u_{1} & 0 \\
0 & \rho c^{2} & 0 & u_{1}
\end{array}\right), \\
B_{2}=\left(\begin{array}{cccc}
u_{2} & 0 & \rho & 0 \\
0 & u_{2} & 0 & 0 \\
0 & 0 & u_{2} & \frac{1}{\rho} \\
0 & 0 & \rho c^{2} & u_{2}
\end{array}\right) .
\end{gathered}
$$

We consider the system with frozen coefficients. Therefore, instead of the theory of pseudodifferential operators, we can use Fourier transformation and some linear algebra calculations to derive the local absorbing boundary conditions. Since this system is not symmetric, we have to apply a well-known transformation [9] to reduce it to a symmetric form. Then, after some matrix multiplication, we can write the system as

$$
\partial_{x} w=A \partial_{t} w+E \partial_{y} w+f
$$

with suitable matrices $A$ and $E$. We apply the Fourier transformation ${ }^{-}$to $w$ with respect to $y$ and $t$ to obtain the following ODE for $\hat{w}$ :

$$
\partial_{x} \hat{w}(x, \omega, s)=M(\omega, s) \hat{w}(x, \omega, s)+\hat{f}(x, \omega, s),
$$

where $M$ is defined as in (12) (see [6]). It turns out that there is a matrix $V(\omega, s)$ such that $V(\omega, s) M(\omega, s) V^{-1}(\omega, s)$ is diagonal. The matrices $V$ and $M$ can be calculated explicitly. When solving this ODE, it can be seen that the incoming waves are related to the eigenvalues of $M(\omega, s)$ with negative imaginary part, and the outgoing ones to those with positive imaginary part. Now the absorbing boundary conditions are such that all incoming waves in $x=0$ are cancelled. This means we have to impose the following boundary conditions:

$$
\pi V \hat{w}=0 \text { for } x=0,
$$

where $\pi$ denotes the projection on those coordinates which are related to the eigenvalues of $M(\omega, s)$ with negative imaginary part. Since these boundary conditions are global for $\hat{w}$, we have to derive a local approximation of them for $w$. As in [2], a second-order approximation is given by

$$
\pi\left(V(0,1) \partial_{t}+\partial_{1} V(0,1) \partial_{y}\right) w=0 \text { for } x=0 .
$$

The values $(\omega, s)=(0,1)$ correspond to waves with normal incidence. Therefore, we have to compute the matrices $V(0,1)$ and $\partial_{1} V(0,1)$. In [2] and [8], this has been done for the symmetric $3 \times 3$ system of the shallow water equations. Engquist and Majda [2] have used Taylor expansion arguments and Wagatha [8] used discrete least squares techniques to get $V(0,1)$ and $\partial_{1} V(0,1)$. In this paper we consider the nonsymmetric $4 \times 4$ system of the linearized Euler 
equations. We shall derive an explicit formula for $V(\omega, s)$, and therefore we can compute $V(0,1)$ and $\partial_{1} V(0,1)$ directly.

In the next section we shall derive the approximate absorbing boundary conditions for a general first-order symmetrizable system with constant coefficients. Then in $\S 3$ we shall apply these results to the system of the Euler equations with frozen coefficients. Finally, in $\S 4$ we shall present numerical results, where we shall use the absorbing boundary conditions as derived in $\S 3$ for the nonlinear Euler equations.

For other concepts of artificial boundary conditions we refer to $[1,4,3,5]$.

\section{ABSORBING BOUNDARY CONDITIONS FOR FIRST-ORDER SYMMETRIZABLE SYSTEMS WITH CONSTANT COEFFICIENTS}

In this section we shall transform a general first-order system with constant coefficients to a decoupled system of ordinary differential equations. In this form it is then obvious how incoming and outgoing waves are related to the eigenvalues of the matrix $M$ (see (4) and (12)). We define the global absorbing boundary condition as in [2] and derive a local first- and second-order approximation thereof.

Let us consider the system

$$
\partial_{t} U+B_{1} \partial_{x} U+B_{2} \partial_{y} U=F \quad \text { in } \mathbf{R}^{2} \times \mathbf{R}^{+}
$$

with initial conditions

$$
U(x, y, 0)=0 \text { in } \mathbf{R}^{2} .
$$

2.1. Assumptions. We assume that $B_{1}, B_{2} \in \mathbf{R}^{n \times n}, B_{1}$ is regular, and that there exists a matrix $T \in \mathbf{R}^{n \times n}$ such that

$$
T^{-1} B_{1} T=\operatorname{diag}\left(\rho_{1}, \ldots, \rho_{n}\right), \quad T^{-1} B_{2} T=\text { symmetric, }
$$

where $\rho_{1}, \ldots, \rho_{n}$ are the eigenvalues of the matrix $B_{1}$. We suppose that there exists a solution $U \in C_{0}^{1}\left(\mathbf{R}^{2} \times \mathbf{R}^{+}\right)$of $(7),(8)$.

Now define $w:=T^{-1} U$ and obtain

$$
\partial_{t} w=A_{1} \partial_{x} w+A_{2} \partial_{y} w+T^{-1} F \quad \text { in } \mathbf{R}^{2} \times \mathbf{R}^{+},
$$

where $A_{1}=-T^{-1} B_{1} T$ and $A_{2}=-T^{-1} B_{2} T$. Then, if we denote $A:=A_{1}^{-1}$, $E:=-A_{1}^{-1} A_{2}$, and $f:=-A_{1}^{-1} T^{-1} F$, equation (10) implies

$$
\partial_{x} w=A \partial_{t} w+E \partial_{y} w+f \quad \text { in } \mathbf{R}^{2} \times \mathbf{R}^{+} \text {. }
$$

Furthermore, we shall use the notation

$$
M(\omega, s):=i s A+i \omega E .
$$

Now we shall investigate the relationship between the eigenvalues of $\lambda A_{1}+\omega A_{2}$ and those of $M(\omega, s)$. Since $\lambda A_{1}+\omega A_{2}$ is symmetric for all $\lambda$ and $\omega$, there exists a set of real eigenvalues $\kappa_{1}(\omega, \lambda), \ldots, \kappa_{n}(\omega, \lambda)$ and a basis of eigenvectors $v_{1}(\omega, \lambda), \ldots, v_{n}(\omega, \lambda)$ of $\lambda A_{1}+\omega A_{2}$. 
2.2. Lemma. Assume that there are real numbers $\lambda_{1}(\omega), \ldots, \lambda_{n}(\omega)$ such that

$$
\kappa_{j}\left(\omega, \lambda_{j}(\omega)\right)=1 \text { for } j=1, \ldots, n \text {, for all } \omega \text {. }
$$

The corresponding eigenvectors are denoted by $r_{j}(\omega):=v_{j}\left(\omega, \lambda_{j}(\omega)\right)$. Then we have

$$
M(\omega, 1) r_{j}(\omega)=i \lambda_{j}(\omega) r_{j}(\omega) .
$$

Proof. By the definition of $\lambda_{j}$ we have

$$
\left(\lambda_{j}(\omega) A_{1}+\omega A_{2}\right) r_{j}=r_{j}
$$

and therefore

$$
\begin{aligned}
A_{1} M(\omega, 1) r_{j}(\omega) & =i A_{1}(A+\omega E) r_{j}=i A_{1}\left(A_{1}^{-1}-\omega A_{1}^{-1} A_{2}\right) r_{j} \\
& =i\left(1-\omega A_{2}\right) r_{j}=i\left(1+\lambda_{j}(\omega) A_{1}-\kappa_{j}\right) r_{j}=i\left(\lambda_{j}(\omega) A_{1}\right) r_{j} .
\end{aligned}
$$

The next lemma gives the eigenvectors of $M(\omega, s)$.

2.3. Lemma. Let us define $\sigma_{j}(\omega, s):=s \lambda_{j}\left(\frac{\omega}{s}\right)$. Then the $r_{j}\left(\frac{\omega}{s}\right)$ are also eigenvectors of $M(\omega, s)$ :

$$
M(\omega, s) r_{j}\left(\frac{\omega}{s}\right)=i \sigma_{j}(\omega, s) r_{j}\left(\frac{\omega}{s}\right) .
$$

Proof. By the definition of $M(\omega, s)$ we get

$$
M(\omega, s) r_{j}\left(\frac{\omega}{s}\right)=s M\left(\frac{\omega}{s}, 1\right) r_{j}\left(\frac{\omega}{s}\right)=i \sigma_{j}(\omega, s) r_{j}\left(\frac{\omega}{s}\right) .
$$

In order to find out the incoming and outgoing modes, we want to apply the Fourier transformation ${ }^{-}$with respect to $t$ and $y$ to the solution $w$ of (11):

$$
\hat{w}(x, \omega, s):=\int_{\mathbf{R}} \int_{\mathbf{R}} e^{-i(s t+\omega y)} w(x, y, t) d y d t .
$$

Here we have extended $w$ to all of $\mathbf{R}^{3}$ by 0 . Therefore, we obtain from (11)

$$
\begin{aligned}
\partial_{x} \hat{w}(x, \omega, s) & =i s A \hat{w}(x, \omega, s)+i \omega E \hat{w}(x, \omega, s)+\hat{f} \\
& =M(\omega, s) \hat{w}(x, \omega, s)+\hat{f} .
\end{aligned}
$$

This is an ODE for $\hat{w}$. In order to get a representation formula for $\hat{w}$, we have to diagonalize the matrix $M(\omega, s)$, using its eigenvectors $r_{j}\left(\frac{\omega}{s}\right)$. Thus, let

$$
V^{-1}(\omega, s):=\left(r_{1}\left(\frac{\omega}{s}\right), \ldots, r_{n}\left(\frac{\omega}{s}\right)\right)
$$

Then we obtain

$$
\begin{aligned}
V(\omega, s) M(\omega, s) V^{-1}(\omega, s) & =\operatorname{diag}\left(i \sigma_{1}(\omega, s), \ldots, i \sigma_{n}(\omega, s)\right) \\
& =: D(\omega, s) .
\end{aligned}
$$

This means we can reduce (18) to the following decoupled system of ODE's for the function $z(x, \omega, s):=V(\omega, s) \hat{w}(x, \omega, s)$ :

$$
\partial_{x} z(x, \omega, s)=D(\omega, s) z(x, \omega, s)+p(x, \omega, s),
$$


where we have used $p(x, \omega, s):=V(\omega, s) \hat{f}(x, \omega, s)$. The solution of $(21)$ can be written as

$$
z_{j}(x, \omega, s):=e^{i \sigma_{j}(\omega, s) x} q_{j}(x, \omega, s)
$$

where

$$
\begin{aligned}
\partial_{x} q_{j}(x, \omega, s) & =e^{-i \sigma_{j}(\omega, s) x} p_{j}(x, \omega, s) \text { and } \\
q_{j}(0, \omega, s) & =(V \hat{w})_{j}(0, \omega, s) .
\end{aligned}
$$

In order to see how $w_{j}$ depends on the eigenvalues $\sigma_{j}$, we express $\hat{w}_{j}$ in terms of $z_{j}$ and apply the inverse Fourier transformation,

$$
\begin{gathered}
\hat{w}_{j}=\left(V^{-1} z\right)_{j}=\sum_{k} V_{j k}^{-1} z_{k}=\sum_{k} V_{j k}^{-1} e^{i \sigma_{k}(\omega, s) x} q_{k}(x, \omega, s), \\
w(x, y, t)_{j}=\sum_{k} \int_{\mathbf{R}} \int_{\mathbf{R}} e^{i\left(s t+\omega y+\sigma_{k}(\omega, s) x\right)} V_{j k}^{-1} q_{k}(x, \omega, s) d \omega d s .
\end{gathered}
$$

Now we would like to show that the terms in (25) can be classified as incoming or outgoing waves. This relationship is used for the definition of the absorbing boundary condition (see [2]). To make this more precise, we have to assume

2.4. Assumption. The eigenvalues $\lambda_{j}(\omega)$ are continuous at $\omega=0$ and

$$
\lambda_{j}(0) \neq 0 \text { for } j=1, \ldots, n \text {. }
$$

2.5. Remark. Consider (7) on $\mathbf{R}^{+} \times \mathbf{R} \times \mathbf{R}^{+}$. The terms in (25) with $\sigma_{k}\left(\frac{\omega}{s}, 1\right) \leq$ 0 are related to incoming waves, and those with $\sigma_{k}\left(\frac{\omega}{s}, 1\right) \geq 0$ to outgoing waves on $\{x=0\}$. The propagation speed of the plane wave

$$
e^{i\left(s t+\omega y+\sigma_{k}(\omega, s) x\right)}
$$

is given by

$$
-\frac{s}{\omega^{2}+\sigma_{k}^{2}}\left(\begin{array}{c}
\sigma_{k} \\
\omega
\end{array}\right) \text {. }
$$

Proof. Let $e^{i c_{0}}$ be the value of the wave (27) at time $t_{0}$ at $\left(x_{0}, y_{0}\right)$ with $c_{0}:=s t_{0}+\omega y_{0}+\sigma_{k}(\omega, s) x_{0}$. Then the wave will obtain the same state $e^{i c_{0}}$ at a later time $t_{1}:=t_{0}+\Delta t$ in $\left(x_{1}, y_{1}\right)$, where $x_{1}$ and $y_{1}$ are defined as follows:

$$
x_{1}=\frac{\left(c_{0}-s t_{1}\right) \sigma_{k}}{\omega^{2}+\sigma_{k}^{2}} \text { and } y_{1}=\frac{\left(c_{0}-s t_{1}\right) \omega}{\omega^{2}+\sigma_{k}^{2}} \text {. }
$$

Then the velocity vector is given by

$$
\frac{1}{\Delta t}\left(\begin{array}{c}
x_{1}-x_{0} \\
y_{1}-y_{0}
\end{array}\right)=-\frac{s}{\omega^{2}+\sigma_{k}^{2}}\left(\begin{array}{c}
\sigma_{k} \\
\omega
\end{array}\right) .
$$

Now it is easy to see that $\operatorname{sign}\left(s \sigma_{k}(\omega, s)\right)=\operatorname{sign} \sigma_{k}\left(\frac{\omega}{s}, 1\right)$. Therefore, we obtain for the velocity vector in the $(x, y)$-coordinate system:

$$
-\frac{1}{\omega^{2}+\sigma_{k}^{2}}\left(\begin{array}{c}
\operatorname{sign}\left(\sigma_{k}\left(\frac{\omega}{s}, 1\right)\right)\left|s \sigma_{k}\right| \\
s \omega
\end{array}\right) .
$$


It is obvious that this velocity vector corresponds to an incoming wave if $\sigma_{j}\left(\frac{\omega}{s}, 1\right) \leq 0$ and to an outgoing wave if $\sigma_{j}\left(\frac{\omega}{s}, 1\right) \geq 0$.

If the boundary conditions are absorbing, then there should be no incoming waves in (25) for $x=0$. This means $V_{j k}^{-1} q_{k}(x, \omega, s)$ should be equal to zero for $k \in\left\{k \mid \sigma_{k}\left(\frac{\omega}{s}, 1\right) \leq 0\right\}$ and $x=0$, that is,

$$
q_{k}(0, \omega, s)=0 \text { for } k \text { such that } \sigma_{k}\left(\frac{\omega}{s}, 1\right) \leq 0 .
$$

This condition can also be written in terms of $\hat{w}$ (see (22)):

$$
(V \hat{w})_{k}(0, \omega, s)=0 \text { for } k \text { such that } \sigma_{k}\left(\frac{\omega}{s}, 1\right) \leq 0 .
$$

We shall use this property for the definition of the exact absorbing boundary condition (see [2]):

2.6. Definition. Condition (33) will be called the exact absorbing boundary condition for the initial value problem (7), (8) in $x=0$.

Since this definition concerns $\hat{w}$, this boundary condition is global. In order to apply it in numerical calculations, we have to derive a local version of it. The idea is to give a local approximation of (33) in the case where we have $|\omega| \leq \omega_{0}$ and $\left|\frac{\omega}{s}\right| \leq \omega_{0}$, where $\omega_{0}$ is sufficiently small. This means we consider only waves with nearly normal incidence at $x=0$ (see (31)). We assume that the following condition is valid.

2.7. Assumption. For all $\omega$ and $s$ such that $|\omega| \leq \omega_{0}$ and $\left|\frac{\omega}{s}\right| \leq \omega_{0}$, with $\omega_{0}$ sufficiently small, we have

$$
V\left(\frac{\omega}{s}, 1\right)=V(0,1)+\frac{\omega}{s} \partial_{1} V(0,1)+O\left(\frac{\omega}{s}\right)^{2} .
$$

Since we have $V(\omega, s)=V\left(\frac{\omega}{s}, 1\right)$, condition (33) implies that for all $k$ such that $\sigma_{k}\left(\frac{\omega}{s}, 1\right) \leq 0$,

$$
\sum_{j}\left(V(0,1)+\frac{\omega}{s} \partial_{1} V(0,1)+O\left(\frac{\omega}{s}\right)^{2}\right)_{k j} \hat{w}_{j}=0 \quad \text { for } x=0 .
$$

As a first-order approximation we obtain

$$
\sum_{j} V(0,1)_{k j} \hat{w}_{j}=0 \text { for } x=0 \text { and for all } k \text { such that } \lambda_{k}(0) \leq 0,
$$

and after applying the inverse Fourier transformation $S$,

$$
\sum_{j} V(0,1)_{k j} w_{j}=0 \text { for } x=0 \text { and } \lambda_{k}(0) \leq 0,
$$

or

$$
\pi_{k}(V(0,1) w)=0 \quad \text { in } x=0 \text { for all } k \text { such that } \lambda_{k}(0) \leq 0 .
$$

Here we have used $\pi_{k}$ to denote the projection on the $k$ th coordinate for which we have $\lambda_{k}(0) \leq 0$. In order to get a second-order approximation, we multiply (35) by $s$ and consider the first two terms only:

$$
\sum_{j}\left(s V(0,1)+\omega \partial_{1} V(0,1)+O\left(\frac{\omega^{2}}{s}\right)\right)_{k j} \hat{w}_{j}=0 .
$$


Again we apply $S$, and (39) implies

$$
\sum_{j}\left(V(0,1)_{k j} S\left(s \hat{w}_{j}\right)+\left(\partial_{1} V(0,1)\right)_{k j} S\left(\omega \hat{w}_{j}\right)\right)=0
$$

or

$$
\sum_{j}\left(V(0,1)_{k j} \partial_{t} w_{j}+\partial_{1} V(0,1)_{k j} \partial_{y} w_{j}\right)=0 \quad \text { in } x=0 \text { and } \lambda_{k}(0) \leq 0 .
$$

This can also be written in the form

$$
\pi_{k}\left(V(0,1) \partial_{t} w+\partial_{1} V(0,1) \partial_{y} w\right)=0 \quad \text { in } x=0 \text { and } \lambda_{k}(0) \leq 0
$$

3. ABSORBING BOUNDARY CONDITIONS FOR THE LINEARIZED EULER EQUATIONS

In this section we shall consider the Euler equation in primitive variables, as described in (1) and (2), with frozen coefficients. First, let us verify Assumption 2.1. For the matrices $T$ and $T^{-1}$ we obtain

$$
T=\left(\begin{array}{cccc}
1 & 0 & \rho / c \sqrt{2} & \rho / c \sqrt{2} \\
0 & 0 & 1 / \sqrt{2} & -1 / \sqrt{2} \\
0 & 1 & 0 & 0 \\
0 & 0 & \rho c / \sqrt{2} & \rho c / \sqrt{2}
\end{array}\right), \quad T^{-1}=\left(\begin{array}{cccc}
1 & 0 & 0 & -1 / c^{2} \\
0 & 0 & 1 & 0 \\
0 & 1 / \sqrt{2} & 0 & 1 / c \rho \sqrt{2} \\
0 & -1 / \sqrt{2} & 0 & 1 / c \rho \sqrt{2}
\end{array}\right) .
$$

It then turns out that

$$
T^{-1} B_{1} T=\left(\begin{array}{cccc}
u_{1} & 0 & 0 & 0 \\
0 & u_{1} & 0 & 0 \\
0 & 0 & u_{1}+c & 0 \\
0 & 0 & 0 & u_{1}-c
\end{array}\right)
$$

where $c:=\sqrt{\gamma p / \rho}$ is the speed of sound. Then, with the notation of $\S 2$, we obtain $A_{1}:=-T^{-1} B_{1} T$ and $A_{2}:=-T^{-1} B_{2} T$,

$$
A_{2}=-\left(\begin{array}{cccc}
u_{2} & 0 & 0 & 0 \\
0 & u_{2} & c / \sqrt{2} & c / \sqrt{2} \\
0 & c / \sqrt{2} & u_{2} & 0 \\
0 & c / \sqrt{2} & 0 & u_{2}
\end{array}\right)
$$

According to (11) we define $A:=A_{1}^{-1}$ and $E:=-A_{1}^{-1} A_{2}$. Now we consider the eigenvalues and eigenvectors of $\lambda A_{1}+\omega A_{2}$.

3.1. Lemma. Let $\omega$ and $\lambda \in \mathbf{R}$ be given, and $\alpha(\omega, \lambda):=\sqrt{\lambda^{2}+\omega^{2}}$. Then the eigenvectors $v_{j}(\omega, \lambda)$ and the eigenvalues $\kappa_{j}(\omega, \lambda)$ of $\lambda A_{1}+\omega A_{2}$ are as follows:

If $\lambda \geq 0$, then

$$
\begin{gathered}
v_{1}=\left(\begin{array}{l}
1 \\
0 \\
0 \\
0
\end{array}\right), \quad v_{2}=\left(\begin{array}{c}
0 \\
\sqrt{2} \lambda \\
-\omega \\
\omega
\end{array}\right), \quad v_{3}=\left(\begin{array}{c}
0 \\
\sqrt{2} \omega \\
\alpha+\lambda \\
\alpha-\lambda
\end{array}\right), \quad v_{4}=\left(\begin{array}{c}
0 \\
-\sqrt{2} \omega \\
\alpha-\lambda \\
\alpha+\lambda
\end{array}\right), \\
\kappa_{1}=-\left(\lambda u_{1}+\omega u_{2}\right), \quad \kappa_{2}=\kappa_{1}, \\
\kappa_{3}=-\left(\lambda u_{1}+\omega u_{2}+\alpha c\right), \quad \kappa_{4}=-\left(\lambda u_{1}+\omega u_{2}-\alpha c\right) .
\end{gathered}
$$


If $\lambda \leq 0$, then

$$
\begin{gathered}
v_{1}=\left(\begin{array}{l}
1 \\
0 \\
0 \\
0
\end{array}\right), \quad v_{2}=\left(\begin{array}{c}
0 \\
\sqrt{2} \lambda \\
-\omega \\
\omega
\end{array}\right), \quad v_{3}=\left(\begin{array}{c}
0 \\
-\sqrt{2} \omega \\
\alpha-\lambda \\
\alpha+\lambda
\end{array}\right), \quad v_{4}=\left(\begin{array}{c}
0 \\
\sqrt{2} \omega \\
\alpha+\lambda \\
\alpha-\lambda
\end{array}\right), \\
\kappa_{1}=-\left(\lambda u_{1}+\omega u_{2}\right), \quad \kappa_{2}=\kappa_{1}, \\
\kappa_{3}=-\left(\lambda u_{1}+\omega u_{2}-\alpha c\right), \quad \kappa_{4}=-\left(\lambda u_{1}+\omega u_{2}+\alpha c\right) .
\end{gathered}
$$

The eigenvectors $v_{j}$ for $j=1, \ldots, 4$ are linearly independent.

These properties can be easily verified.

3.2. Remark. We have to distinguish between the cases $\lambda \geq 0$ and $\lambda \leq 0$ in order to be consistent with the labeling of the eigenvalues of $\lambda A_{1}+\omega A_{2}$ if $\omega=0$ and $\lambda=1$. This can be seen as follows. If we use (45), (46), (47) for $\lambda \geq 0$ as well as for $\lambda \leq 0$, we would get, for instance for $\kappa_{3}$,

$$
\kappa_{3}=-\left(\lambda u_{1}+\alpha c\right)=-\left(\lambda u_{1}+|\lambda| c\right) \text {. }
$$

Assume that $\kappa_{3}=1$. Then we know from Lemma 2.2 that the corresponding $\lambda$ is an eigenvalue of $-i M(0,1)$. Formula (51) then implies that

$$
\lambda=-\frac{1}{u_{1}+c} \text { if } \lambda \geq 0
$$

and

$$
\lambda=-\frac{1}{u_{1}-c} \text { if } \lambda \leq 0 .
$$

But since $-i M(0,1)=A$, the third eigenvalue of $-i M(0,1)$ should be equal to $-1 /\left(u_{1}+c\right)$, independent of the sign of $\lambda$.

In order to get the eigenvalues of $M(\omega, 1)$, we have to solve the equations (13). They are quadratic in $\lambda$. We obtain the following result.

3.3. Lemma. Let $\beta:=-\omega u_{2}-1, \omega \in \mathbf{R}$, and $u_{1} \pm c \neq 0$. Then the eigenvalues of $-i M(\omega, 1)$ are given by

$$
\begin{gathered}
\lambda_{1}(\omega):=\frac{\beta}{u_{1}}, \quad \lambda_{2}(\omega):=\lambda_{1}(\omega), \\
\lambda_{3}(\omega):=\frac{u_{1} \beta}{u_{1}^{2}-c^{2}}+\frac{c}{u_{1}^{2}-c^{2}} \sqrt{\beta^{2}+\omega^{2}\left(u_{1}^{2}-c^{2}\right)}, \\
\lambda_{4}(\omega):=\frac{u_{1} \beta}{u_{1}^{2}-c^{2}}-\frac{c}{u_{1}^{2}-c^{2}} \sqrt{\beta^{2}+\omega^{2}\left(u_{1}^{2}-c^{2}\right)} .
\end{gathered}
$$

In particular, according to Remark 3.2, we have

$$
\lambda_{3}(0)=-\frac{1}{u_{1}+c} \text { and } \lambda_{4}(0)=-\frac{1}{u_{1}-c} .
$$

Furthermore, these values satisfy Assumption 2.4. 
Proof. First of all, it is obvious that the $\lambda_{i}(\omega)$ are continuous in $\omega$ and that they satisfy (52). Therefore, Assumption 2.4 is valid. In Lemma 2.2 we have shown that the eigenvalues of $-i M(\omega, 1)$ are given by the solution of

$$
\kappa_{j}\left(\omega, \lambda_{j}(\omega)\right)=1 \text {, }
$$

where the $\kappa_{j}$ are defined in (46), (47) and (49), (50), respectively. For $\lambda_{1}(\omega)$ and $\lambda_{2}(\omega)$, the condition (13) is satisfied. Now let us verify (13) for $\lambda_{3}(\omega)$ and $\lambda_{4}(\omega)$. For $i=3,4$ we obtain

$$
\left(\lambda_{i}(\omega)-\frac{u_{1} \beta}{u_{1}^{2}-c^{2}}\right)^{2}=\frac{c^{2}}{\left(u_{1}^{2}-c^{2}\right)^{2}}\left(\beta^{2}+\omega^{2}\left(u_{1}^{2}-c^{2}\right)\right) .
$$

After some elementary calculations we get

$$
\left|\lambda_{i} u_{1}+\omega u_{2}+1\right|=c|\alpha|=c \alpha .
$$

Assume first that $i=3$ and $\lambda_{3}(0)>0$. Then by continuity we also have

$$
\begin{aligned}
\lambda_{3}(\omega) u_{1}+\omega u_{2}+1 & =\left(\lambda_{3}(\omega)-\lambda_{3}(0)\right) u_{1}+\omega u_{2}+1+\lambda_{3}(0) u_{1} \\
& =\left(\lambda_{3}(\omega)-\lambda_{3}(0)\right) u_{1}+\omega u_{2}-c \lambda_{3}(0)<0
\end{aligned}
$$

for $\omega$ sufficiently small. Therefore, (53) implies

$$
\lambda_{3} u_{1}+\omega u_{2}+1=-c \alpha,
$$

and therefore (47). Similarly we obtain for $i=3$ and $\lambda_{3}(0)<0$ the condition (50). The statements concerning $i=4$ can be proved in the same way.

According to Lemma 2.2, the eigenvectors $r_{j}(\omega)$ of $M(\omega, 1)$ are then given by $r_{j}(\omega)=v_{j}\left(\omega, \lambda_{j}(\omega)\right)$. In the following we shall use the notation $s_{j}=$ $\operatorname{sign}\left(\lambda_{j}\right)$. For small $\omega$ this is independent of $\omega$. We obtain

$$
\begin{array}{cc}
r_{1}=\left(\begin{array}{l}
1 \\
0 \\
0 \\
0
\end{array}\right), & r_{2}=\left(\begin{array}{c}
0 \\
\sqrt{2} \lambda_{2} \\
-\omega \\
\omega
\end{array}\right), \\
r_{3}=\left(\begin{array}{c}
0 \\
\sqrt{2} s_{3} \omega \\
\alpha_{3}+\left|\lambda_{3}\right| \\
\alpha_{3}-\left|\lambda_{3}\right|
\end{array}\right), \quad r_{4}=\left(\begin{array}{c}
0 \\
-\sqrt{2} s_{4} \omega \\
\alpha_{4}-\left|\lambda_{4}\right| \\
\alpha_{4}+\left|\lambda_{4}\right|
\end{array}\right) .
\end{array}
$$

Let $l_{j}(\omega)$ be defined such that $\left|l_{i}^{t} r_{j}\right|=\left|l_{i}^{t} r_{j}\right| \delta_{i j}$. We obtain

$$
\begin{gathered}
l_{1}=\left(\begin{array}{l}
1 \\
0 \\
0 \\
0
\end{array}\right), \quad l_{2}=\left(\begin{array}{c}
0 \\
\sqrt{2} \lambda_{2} u_{1} \\
-\omega\left(u_{1}+c\right) \\
\omega\left(u_{1}-c\right)
\end{array}\right), \\
l_{3}=\left(\begin{array}{c}
0 \\
\sqrt{2} s_{3} \omega u_{1} \\
\left(\alpha_{3}+\left|\lambda_{3}\right|\right)\left(u_{1}+c\right) \\
\left(\alpha_{3}-\left|\lambda_{3}\right|\right)\left(u_{1}-c\right)
\end{array}\right), \quad l_{4}=\left(\begin{array}{c}
-\sqrt{2} s_{4} \omega u_{1} \\
\left(\alpha_{4}-\left|\lambda_{4}\right|\right)\left(u_{1}+c\right) \\
\left(\alpha_{4}+\left|\lambda_{4}\right|\right)\left(u_{1}-c\right)
\end{array}\right) .
\end{gathered}
$$


This will be proved in Lemma 3.4. Let $\tau_{j}=\left(l_{j}^{t} r_{j}\right)^{-1}$. Then we define $V^{*}(\omega, 1)$ as

$$
V^{*}(\omega, 1)=\left(r_{1} \tau_{1}, r_{2} \tau_{2}, r_{3} \tau_{3}, r_{4} \tau_{4}\right) .
$$

3.4. Lemma. Let $V(\omega, 1)$ be

$$
V(\omega, 1)=\left(l_{1}, l_{2}, l_{3}, l_{4}\right)^{t} .
$$

Then $V^{-1}(\omega, 1)=V^{*}(\omega, 1)$, and $V(\omega, 1)$ satisfies Assumption 2.7.

Proof. The differentiability of $\lambda_{i}(\omega)$ with respect to $\omega$ for small $\omega$ (see Lemma 3.3) implies that $V$ satisfies Assumption 2.7. Since $V^{*}(\omega, 1)=\left(r_{1} \tau_{1}, r_{2} \tau_{2}\right.$, $\left.r_{3} \tau_{3}, r_{4} \tau_{4}\right)$ and $V(\omega, 1)=\left(l_{1}, l_{2}, l_{3}, l_{4}\right)^{t}$, the definition of $\tau_{i}$ implies that the diagonal elements of $V V^{*}$ are equal to 1 . Then it remains to show that $l_{i}^{t} r_{j}=0$ for $i \neq j$. We shall prove that $l_{3}^{t} r_{2}=0$ and $l_{3}^{t} r_{4}=0$. The other cases are treated similarly. We have

$$
\begin{aligned}
l_{3} r_{2} & =2 \lambda_{2} s_{3} \omega u_{1}-\omega\left(\alpha_{3}+\left|\lambda_{3}\right|\right)\left(u_{1}+c\right)+\omega\left(\alpha_{3}-\left|\lambda_{3}\right|\right)\left(u_{1}-c\right) \\
& =2 \lambda_{2} s_{3} \omega u_{1}-2 \omega\left(\alpha_{3} c+\left|\lambda_{3}\right| u_{1}\right) .
\end{aligned}
$$

If $\lambda_{3}>0$, according to (47) with $\kappa_{3}=1$, the term on the far right in (60) is equal to

$$
2 \lambda_{2} \omega u_{1}-2 \omega\left(-1-\omega u_{2}\right)=0 .
$$

If $\lambda_{3}<0$, according to (50) with $\kappa_{3}=1$, the same term in (60) is equal to

$$
-2 \lambda_{2} \omega u_{1}-2 \omega\left(1+\omega u_{2}\right)=0 \text {. }
$$

For $l_{3}^{t} r_{4}$ we obtain

$$
\begin{aligned}
l_{3} r_{4}= & -2 \omega^{2} s_{3} s_{4} u_{1}+\left(\alpha_{4}-\left|\lambda_{4}\right|\right)\left(\alpha_{3}+\left|\lambda_{3}\right|\right)\left(u_{1}+c\right) \\
& +\left(\alpha_{4}+\left|\lambda_{4}\right|\right)\left(\alpha_{3}-\left|\lambda_{3}\right|\right)\left(u_{1}-c\right) \\
= & -2 \omega^{2} s_{3} s_{4} u_{1}+2 u_{1}\left(\alpha_{3} \alpha_{4}-\left|\lambda_{3} \lambda_{4}\right|\right)+2 c\left(\alpha_{4}\left|\lambda_{3}\right|-\alpha_{3}\left|\lambda_{4}\right|\right) .
\end{aligned}
$$

First assume that $\lambda_{3} \geq 0$ and $\lambda_{4} \geq 0$. Then we have

$$
l_{3}^{t} r_{4}=-2 \omega^{2} u_{1}+2 u_{1}\left(\alpha_{3} \alpha_{4}-\lambda_{3} \lambda_{4}\right)+2 c\left(\alpha_{4} \lambda_{3}-\alpha_{3} \lambda_{4}\right) \text {. }
$$

Using

$$
\beta=-1-\omega u_{2}, \quad \alpha_{3} c=\beta-\lambda_{3} u_{1}, \quad \alpha_{4} c=-\beta+\lambda_{4} u_{1},
$$

from the definition of $\lambda_{3}$ and $\lambda_{4}$ we obtain

$$
\alpha_{3} \alpha_{4}-\lambda_{3} \lambda_{4}=\frac{1}{c^{2}}\left(-\beta^{2}+\beta u_{1}\left(\lambda_{4}+\lambda_{3}\right)-\lambda_{3} \lambda_{4} u_{1}^{2}-\lambda_{3} \lambda_{4} c^{2}\right)
$$

and

$$
\alpha_{4} \lambda_{3}-\alpha_{3} \lambda_{4}=\frac{1}{c}\left(-\beta\left(\lambda_{4}+\lambda_{3}\right)+2 \lambda_{3} \lambda_{4} u_{1}\right)
$$


Therefore,

$$
\begin{aligned}
l_{3}^{t} r_{4} & =-2 \omega^{2} u_{1}+\frac{2 \beta}{c^{2}}\left(\lambda_{3}+\lambda_{4}\right)\left(u_{1}^{2}-c^{2}\right)-\frac{2 u_{1}}{c^{2}}\left(\beta^{2}+\lambda_{3} \lambda_{4}\left(u_{1}^{2}-c^{2}\right)\right) \\
& =-2 \omega^{2} u_{1}+\frac{4 \beta}{c^{2}} u_{1} \beta-\frac{2 u_{1}}{c^{2}}\left(\beta^{2}+\frac{u_{1}^{2} \beta^{2}}{\left(u_{1}^{2}-c^{2}\right)}-\frac{c^{2} \beta^{2}}{\left(u_{1}^{2}-c^{2}\right)}-c^{2} \omega^{2}\right) \\
& =0
\end{aligned}
$$

Now if $\lambda_{3} \leq 0$ and $\lambda_{4} \geq 0$, we have

$$
\beta=-1-\omega u_{2}, \quad \alpha_{3} c=-\beta+\lambda_{3} u_{1}, \quad \alpha_{4} c=-\beta+\lambda_{4} u_{1},
$$

and therefore

$$
\alpha_{3} \alpha_{4}-\left|\lambda_{3} \lambda_{4}\right|=\frac{1}{c^{2}}\left(\beta^{2}-\beta u_{1}\left(\lambda_{4}+\lambda_{3}\right)+\lambda_{3} \lambda_{4} u_{1}^{2}+\lambda_{3} \lambda_{4} c^{2}\right)
$$

and

$$
\alpha_{4}\left|\lambda_{3}\right|-\alpha_{3}\left|\lambda_{4}\right|=\frac{1}{c}\left(\beta\left(\lambda_{4}+\lambda_{3}\right)-2 \lambda_{3} \lambda_{4} u_{1}\right) .
$$

Then we can continue as in (65). The other cases are treated similarly.

We are now able to evaluate the first- and second-order approximating local boundary conditions (38) and (42). The first-order boundary condition (38) for $U$ can be written as

$$
\pi_{k}\left(V(0,1) T^{-1} U\right)=0 \text { for } x=0 \text { and } \lambda_{k}(0) \leq 0 .
$$

From (59) we obtain for $V(0,1) T^{-1}$

$$
V(0,1) T^{-1}=\left(\begin{array}{cccc}
1 & 0 & 0 & -1 / c^{2} \\
0 & 0 & -\sqrt{2} & 0 \\
0 & -s_{3} \sqrt{2} & 0 & -\sqrt{2} s_{3} / \rho c \\
0 & s_{4} \sqrt{2} & 0 & -\sqrt{2} s_{4} / \rho c
\end{array}\right) .
$$

Therefore, (38) implies

3.5. First-order absorbing boundary condition in $x=0$ :

$$
\begin{aligned}
& \text { if } \lambda_{1} \leq 0: \quad \rho-\frac{1}{c^{2}} p=0, \\
& \text { if } \lambda_{2} \leq 0: \quad u_{2}=0, \\
& \text { if } \lambda_{3} \leq 0: u_{1}+\frac{1}{\rho c} p=0, \\
& \text { if } \lambda_{4} \leq 0: u_{1}-\frac{1}{\rho c} p=0 .
\end{aligned}
$$

For calculating the second-order approximating local absorbing boundary condition $(42)$, we need $\partial_{1} V(0,1)$ and therefore $\partial_{\omega} \lambda_{j}$. From Lemma 3.3 
we obtain

$$
\begin{gathered}
\partial_{\omega} \lambda_{1}(0)=-\frac{u_{2}}{u_{1}}, \quad \partial_{\omega} \lambda_{2}(0)=-\frac{u_{2}}{u_{1}} \\
\partial_{\omega} \lambda_{3}(0)=-\frac{u_{2}}{u_{1}+c} \\
\partial_{\omega} \lambda_{4}(0)=-\frac{u_{2}}{u_{1}-c} .
\end{gathered}
$$

Using $\partial_{\omega} \alpha_{j}(0)=\operatorname{sign}\left(\lambda_{j}(0)\right) \partial_{\omega} \lambda_{j}(0)$, we get

(75) $\partial_{1} V(0,1)=\left(\begin{array}{cccc}0 & 0 & 0 & 0 \\ 0 & -\sqrt{2} u_{2} & -\left(u_{1}+c\right) & \left(u_{1}-c\right) \\ 0 & \sqrt{2} u_{1} s_{3} & 2 s_{3} \partial_{\omega} \lambda_{3}(0)\left(u_{1}+c\right) & 0 \\ 0 & -\sqrt{2} u_{1} s_{4} & 0 & 2 s_{4} \partial_{\omega} \lambda_{4}(0)\left(u_{1}-c\right)\end{array}\right)$.

Now the matrices $V(0,1)$ and $\partial_{1} V(0,1)$ are available, and we can compute the approximating absorbing boundary conditions as defined in (42). Since $w=T^{-1} U$, we get

$$
\pi_{k}\left(V(0,1) T^{-1} \partial_{t} U+\partial_{1} V(0,1) T^{-1} \partial_{y} U\right)=0 \text { in } x=0 \text { and } \lambda_{k}(0) \leq 0 .
$$

For $\partial_{1} V(0,1) T^{-1}$ we obtain

$$
\partial_{1} V(0,1) T^{-1}=\left(\begin{array}{cccc}
0 & 0 & 0 & 0 \\
0 & -\sqrt{2} u_{1} & -\sqrt{2} u_{2} & -\sqrt{2} / \rho \\
0 & -s_{3} \sqrt{2} u_{2} & s_{3} \sqrt{2} u_{1} & -\sqrt{2} s_{3} u_{2} / \rho c \\
0 & s_{4} \sqrt{2} u_{2} & -s_{4} \sqrt{2} u_{1} & -\sqrt{2} s_{4} u_{2} / \rho c
\end{array}\right)
$$

Now (42) implies the following

3.6. Second-order absorbing boundary conditions in $x=0$ :

$$
\begin{array}{rlrl}
\text { if } \lambda_{1} \leq 0: & \partial_{t} \rho-\frac{1}{c^{2}} \partial_{t} p=0 \\
\text { if } \lambda_{2} \leq 0: & \partial_{t} u_{2}+u_{1} \partial_{y} u_{1}+u_{2} \partial_{y} u_{2}+\frac{1}{\rho} \partial_{y} p=0, \\
\text { if } \lambda_{3} \leq 0: & \partial_{t} u_{1}+\frac{1}{\rho c} \partial_{t} p+u_{2} \partial_{y} u_{1}-u_{1} \partial_{y} u_{2}+\frac{u_{2}}{\rho c} \partial_{y} p=0, \\
\text { if } \lambda_{4} \leq 0:-\partial_{t} u_{1}+\frac{1}{\rho c} \partial_{t} p-u_{2} \partial_{y} u_{1}+u_{1} \partial_{y} u_{2}+\frac{u_{2}}{\rho c} \partial_{y} p=0
\end{array}
$$

\section{NUMERICAL RESULTS}

The boundary conditions 3.5, 3.6 are implemented in the following way. At each point we have to decide if $\lambda_{j}$ is nonnegative for $j=1, \ldots, 4$. Then the corresponding boundary conditions are discretized explicitly in time and with upwind differences in space. For those values which are not defined by the boundary conditions (78), we use an extrapolation formula of second order to extend them up to the boundary.

We have used these local absorbing boundary conditions for $U$ in some numerical test problems. In our example we consider the nonlinear Euler equations 


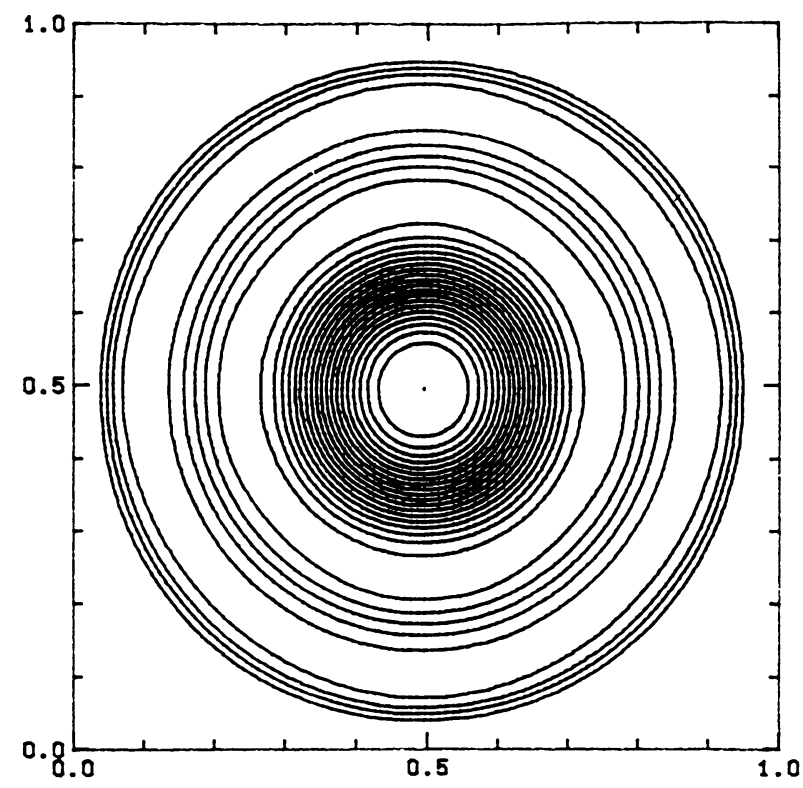

FIGURE 1

as described in (1) and (2) with respect to piecewise constant initial values. Outside of a given circle $C$ we prescribe $U=\left(\rho, u_{1}, u_{2}, p\right)=(1.0,0.0,0.0,0.4)$, and inside of it $U=\left(\rho, u_{1}, u_{2}, p\right)=(4.0,0.0,0.0,1.6)$. The solution will consist of a rotationally symmetric shock wave running outside, a contact discontinuity following the shock wave, and a rarefaction wave going to the center. On the left vertical part of the boundary we impose the absorbing boundary conditions 3.5 and 3.6, respectively. For the discretization of the Euler equation we have used the Harten-Lax-v.Leer scheme (see [7]). The calculations are performed on a $100 \times 100$ grid.

The level lines of the density have been plotted in Figure 1 after 30 time steps. Up to this time, the shock wave has not reached the boundaries. Then, in a second experiment we have shifted the center of the circle $C$ to the left such that the shock front will reach the left boundary within 30 time steps. On the boundary $x=0$ we now imposed the local absorbing boundary conditions 3.6. The level lines of the density are plotted in Figure 2. It can be seen that the solution crosses the boundary nearly without any reflections. For comparison, we have plotted the same situation in Figure 3 but now with reflecting boundary conditions on $x=0$.

Since the absorbing boundary conditions 3.5 and 3.6 are only approximations, there will be small reflected waves. In order to measure the reflected part, we subtract the shifted solution from the original one and calculate the $L^{2}$ norm of the difference. Let the upper indices 1 and 2 refer to the differences corresponding to the absorbing boundary conditions of first and second order, 


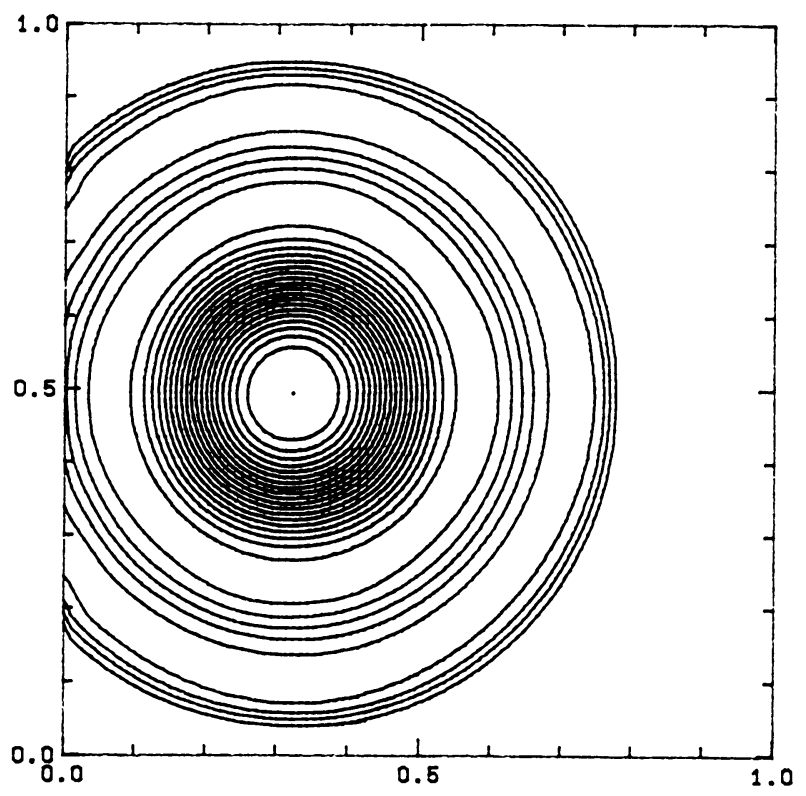

FiguRE 2

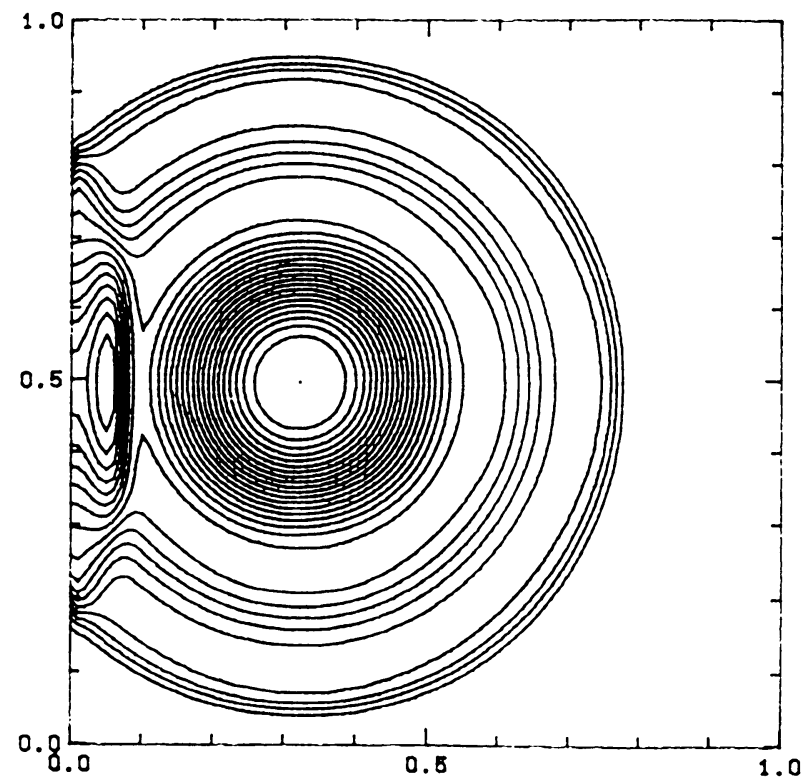

Figure 3 
respectively. Then we obtain

$$
\begin{aligned}
\left\|\rho^{1}\right\|_{L^{2}} & =0.134, & & \left\|\rho^{2}\right\|_{L^{2}}=0.003, \\
\left\|u_{1}^{1}\right\|_{L^{2}} & =0.06, & & \left\|u_{1}^{2}\right\|_{L^{2}}=0.0008, \\
\left\|u_{2}^{1}\right\|_{L^{2}} & =0.007, & & \left\|u_{2}^{2}\right\|_{L^{2}}=0.0007, \\
\left\|p^{1}\right\|_{L^{2}} & =0.03, & & \left\|p^{2}\right\|_{L^{2}}=0.0007 .
\end{aligned}
$$

\section{ACKNOWLEDGMENT}

I thank Dr. U. Thiel, who has improved the implementation of the HartenLax-v.Leer scheme.

\section{BIBLIOGRAPHY}

1. A. Bayliss and E. Turkel, Outflow boundary conditions for fluid dynamics, J. Comput. Phys. 48 (1982), 182-199.

2. B. Engquist and A. Majda, Absorbing boundary conditions for the numerical simulation of waves, Math. Comp. 31 (1977), 629-651.

3. L. Ferm, Open boundary conditions for external flow problems, preprint, 1990.

4. L. Ferm and B. Gustafsson, A downstream boundary procedure for the Euler equations, Comput. Fluids 10 (1982), 261-276.

5. B. Gustafsson, Inhomogeneous conditions at open boundaries for wave propagation problems, Appl. Numer. Math. 4 (1988), 3-19.

6. R. L. Higdon, Initial-boundary value problems for linear hyperbolic systems, SIAM Rev. 28 (1986), 177-217.

7. A. Harten, P. Lax, and B. van Leer, On upstream differencing and Godunov-type schemes for the hyperbolic conservation laws, SIAM Rev. 25 (1983), 35-60.

8. L. Wagatha, Approximation of pseudodifferential operators in absorbing boundary conditions for hyperbolic equations, Numer. Math. 42 (1983), 52-64.

9. R. F. Warming, R. M. Beam, and B. J. Hyett, Diagonalization and simultaneous symmetrization of the gas-dynamic matrices, Math. Comp. 29 (1975), 1037-1045.

INSTitut Für ANGEWANdte Mathematik, Universität Heidelberg, IM NeUenheimer Feld 294, D-6900 Heidelberg, Federal Republic of Germany

E-mail address: ap5@dhdurz2.bitnet 